This item was submitted to Loughborough's Research Repository by the author.

Items in Figshare are protected by copyright, with all rights reserved, unless otherwise indicated.

\title{
Ways of being strands: cross-disciplinary collaboration using craft and mathematics
}

\section{PLEASE CITE THE PUBLISHED VERSION}

https://doi.org/10.1162/DESI_a_00461

\section{PUBLISHER}

(C) Massachusetts Institute of Technology (MIT Press)

\section{VERSION}

AM (Accepted Manuscript)

\section{PUBLISHER STATEMENT}

This work is made available according to the conditions of the Creative Commons Attribution-NonCommercialNoDerivatives 4.0 International (CC BY-NC-ND 4.0) licence. Full details of this licence are available at: https://creativecommons.org/licenses/by-nc-nd/4.0/

\section{LICENCE}

CC BY-NC-ND 4.0

\section{REPOSITORY RECORD}

Nimkulrat, Nithikul, and Janette Matthews. 2019. "Ways of Being Strands: Cross-disciplinary Collaboration Using Craft and Mathematics". figshare. https://hdl.handle.net/2134/24429. 


\title{
Ways of Being Strands: Cross-Disciplinary
}

\section{Collaboration using Craft and Mathematics}

\author{
Nithikul Nimkulrat, Janette Matthews
}

\section{Introduction: Mathematics, Craft, and Textiles}

The intersection of mathematics and craft $^{1}$ can be seen through previous explorations. Mathematicians have noticed the potential for the expression of mathematical concepts through craft media using artifacts as a means of making abstract mathematics explicit. Textile and other craft techniques may be used to transform a complex mathematical idea into a material object in order to demonstrate proof of concept and also to facilitate the understanding of both the mathematics and the craft. This simultaneously provides textile practitioners with insights into the underlying mathematics. Conversely, mathematicians utilizing mathematical methods may analyze art and design works made by textile craft techniques and offer an inspiring perspective on the creation of new designs. Crocheting, knitting, and

\footnotetext{
${ }^{1}$ In this article, craft is used to identify neither a discipline in its own right nor a sub-discipline of art or design, but a method of logically and intellectually thinking through the hand manipulating a material that is utilized in disciplines such as textiles, ceramics, etc. For further discussion about the definition of craft, see Nithikul Nimkulrat, "Material inspiration: From Practice-Led Research to Craft Art Education," Craft Research 1, no. 1 (2010): 63-84; Nithikul Nimkulrat, "Hands-on Intellect: Integrating Craft Practice into Design Research," International Journal of Design 6, no. 3 (2012): 1-14; Carole Gray and Gordon Burnett, "Making Sense: An Exploration of Ways of Knowing Generated Through Practice and Reflection in Craft," in Crafticulation and Education, ed. L. K. Kaukinen (Helsinki: NordFo, 2009), 44-51; and Richard Sennett, The Craftsman (New Haven, CT: Yale University Press, 2008).
} 
weaving are textile craft techniques that mathematicians often use to explore and communicate wide-ranging mathematics including topology, geometry, etc. Through crocheting, the Lorenz manifold's computer-generated images are transformed into a textile piece that explicitly conveys its properties, ${ }^{2}$ while the concept of hyperbolic geometry is illustrated through crocheted models. ${ }^{3}$ The topological principles of knitting have been studied, leading to the invention of two new homogeneous methods of knitting Mobius strips. ${ }^{4}$ In weaving, tessellation theory, computational tools, and laser cutting have been employed to examine the houndstooth pattern, resulting in a new weaving pattern. ${ }^{5}$ Other studies examine the mathematical content of various textile activities and illustrate how skills in mathematics may be acquired through the learning of textile crafts. ${ }^{6}$

${ }^{2}$ Hinke M. Osinga and Bernd Krauskopf, "Crocheting the Lorenz Manifold," The Mathematical Intelligencer 26, no. 4 (2004): 25-37 and Hinke M. Osinga and Bernd Krauskopf, "How to Crochet a Space-Filling Pancake: The Math, The Art and What Next," in Bridges 2014: Mathematics, Music, Art, Architecture, Culture, ed. Gary Greenfield, George W. Hart, and Reza Sarhangi (Phoenix, Az: Tessellations, 2014): 19-26.

${ }^{3}$ Diana Taimina, Crocheting Adventures with Hyperbolic Planes (Wellesley, MA: AK Peters, 2009).

${ }^{4}$ Daniel C. Isaksen and Alabama P. Petrofsky, "Mobius Knitting," in Bridges 1999: Mathematical Connections in Art, Music, and Science, ed. Reza Sarhangi (Arkansas, KS: Gilliland, 1999): 67-76.

${ }^{5}$ Loe M. G. Feijs, "Geometry and Computation of Houndstooth (Pied-de-Poule)," in Bridges 2012: Mathematics, Music, Art, Architecture, Culture, ed. Robert Bosch, Douglas McKenna, and Reza Sarhangi (Phoenix, Az: Tessellations, 2012): 299-306.

${ }^{6}$ For example, the issues of gender are tackled in the agendas of mathematics education through an exploration of how textile activities and their mathematical content can be used in the teaching of mathematics—see Mary Harris, "Common Threads: Mathematics and Textiles," Mathematics in School 17, no. 4 (1988): 24-28 and Mary Harris, Common Threads: Women, Mathematics and Work (Stokeon-Trent: Trentham Books, 1997). Another area of study is concerned with the exploration of how mathematical concepts can be crafted with the help of fiber arts-see Sarah-Marie Belcastro, and Carolyn Yackel, ed., Making Mathematics with Needlework: Ten Papers and Ten Projects (Natick, MA: 
These examples present mathematics research exploring connections between mathematics and textiles conducted by mathematicians who, within their research, may act as non-professional designers or crafters and perform their research through craft. As the connections between the two disciplines are studied from mathematicians' or scientists' viewpoints, the results of their research that may be useful for the design of textiles are communicated in the language of mathematics. This is problematic as it is a language that designers may not understand as generally they may not have had a formal mathematics training and both concepts and notation are inaccessible. More recently, collaborative research between mathematicians and professional designers has begun to emerge. For example, collaborative work between a fashion designer and a mathematician in applying fractals to examine basic fabric construction has led to the development of new pattern designs: a pattern for laser cutting derived from the houndstooth weave ${ }^{7}$ and one for digital printing derived from warp knitting. ${ }^{8}$ Similarly, the 1325 ISSEY MIYAKE series is the result of a collaboration to explore the mathematics of folding. ${ }^{9}$ This cross-disciplinary ${ }^{10}$ collaboration between the mathematician and the designer seems to be a key approach to overcome the issues with mathematical

A K Peters, 2009) and Sarah-Marie Belcastro, and Carolyn Yackel ed., Crafting by Concepts: Fiber Arts and Mathematics (Natick, MA: A K Peters, 2011).

${ }^{7}$ Loe M. G. Feijs and Marina Toeters, "Constructing and Applying the Fractal Pied de Poule (Houndstooth)," in Bridges 2013: Mathematics, Music, Art, Architecture, Culture, ed. George W. Hart and Reza Sarhangi (Phoenix, Az: Tessellations, 2013): 429-32.

${ }^{8}$ Loe M. G. Feijs, Marina Toeters, Jun Hu, and Jihong Liu, "Design of a Nature-Like Fractal Celebrating Warp Knitting," in Bridges 2014: Mathematics, Music, Art, Architecture, Culture, ed. Gary Greenfield, George W. Hart, and Reza Sarhangi (Phoenix, Az: Tessellations, 2014): 369-72.

${ }^{9}$ See http://www.isseymiyake.com/en/brands/132_5.html.

${ }^{10}$ The notion of "cross-disciplinary" in this article is used as the overarching term to indicate practices that concern more than one discipline, including "a collection of practices such as multidisciplinary, 
language and to successfully make research into this area understandable by designers. The account of ongoing research presented in this article supports this approach. This article builds on and extends previous published work on a collaborative project investigating the relationship between mathematical knot theory and knotted textiles. ${ }^{11}$ It highlights how cross-disciplinary collaboration can bring knowledge from one discipline, i.e., mathematics, to provoke research and practice in another, i.e., craft/design.

The project originated when the first author, Nimkulrat, a designer-researcher who has used knotting as a technique for creating textiles for a decade, came across diagrams from knot theory that appeared as if they were visualizations of her knotted structures. Although Nimkulrat studied mathematics at school and university, this did not include topology, a branch of mathematics concerned with the properties of space that are preserved under continuous transformations, such as stretching, twisting, and bending. ${ }^{12}$ To investigate possible links between the observed knot diagrams, supporting mathematical theory, and physical textiles, it was necessary for Nimkulrat to seek a collaborator who had the appropriate mathematical knowledge. A conversation was initiated with Matthews, the second author, who is a textile design-

interdisciplinary, and transdisciplinary" that involve "negotiating among different perspectives and territories of expertise, and innovation and transformative processes." Robin Adams, "Cross-Disciplinary Ways of Thinking, Acting, and Being" (lecture, Tufts University, Medford, MA, December 6, 2010), http://sites.tufts.edu/tuftsstemlecture/previous-lectures/cross-disciplinary-ways-of-thinking-acting-andbeing/.

\footnotetext{
${ }^{11}$ Nithikul Nimkulrat and Janette Matthews, "Ways of Being Strands: Exploration of Textile Craft Knots by Hand and Mathematics," in Proceedings of the 2nd Biennial Research Through Design Conference, 25-27 March 2015, Cambridge, UK, Article 14, DOI: 10.6084/m9.figshare.1327996.

${ }^{12}$ Discontinuous transformations, such as cutting and puncturing, are not permitted. See Fred $\mathrm{H}$.

Croom, Principles of Topology (Mineola, NY: Dover Publications, 2016): 1-4.
} 
researcher with a background in mathematics. ${ }^{13}$ The authors' areas of mutual interest were explored which led to the ongoing research collaboration. Research questions include: (1) whether craft and mathematical knots share comparable characteristics, (2) whether knot theory can examine the mathematical properties of knotted textile structures, and (3) how knot theory can facilitate the design and production of knotted textiles. This research aimed to contribute to existing work investigating connections between mathematics and textiles. An artifact (Figure 1a) was created as part of the process of enquiry to illustrate how knot theory may inform and promote the design of knotted textiles.

\footnotetext{
${ }^{13}$ Matthews studied natural sciences majoring in pure and applied mathematics (1976-80). She then studied textile design (1996-2000) completing a cross-disciplinary PhD in 2011. She has worked in mathematical education supporting students in their undergraduate learning of mathematics and currently lectures in textile design. She has a research interest in the communication of mathematics to art and design practitioners who, whilst often interested in mathematical concepts, are excluded by prior learning.
} 

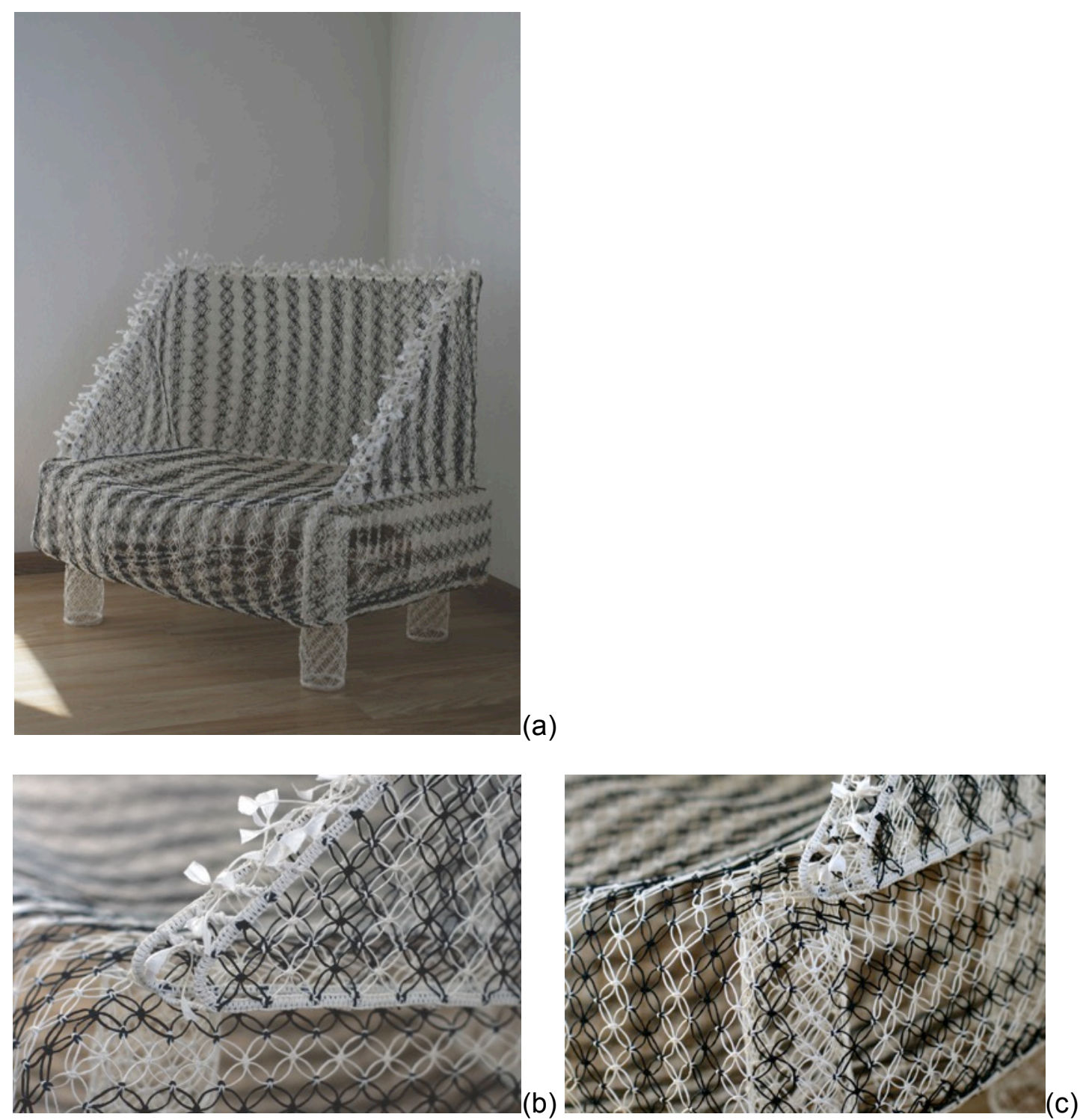

Figure 1. "Black \& White Striped Armchair" (2014) (a) and its details (b and c), dimensions: $62 \mathrm{~cm} \mathrm{x}$ $50 \mathrm{~cm} \times 63 \mathrm{~cm}$, weight $1.5 \mathrm{~kg}$, material: paper string. (C) Nithikul Nimkulrat.

\section{Mathematical Knot Theory and Craft Knots}

Responding to the research questions requires both conducting cross-disciplinary research through design practice ${ }^{14}$ and understanding the basics of mathematical knot theory. Knot theory is a subset of mathematical topology that studies properties

\footnotetext{
${ }^{14}$ Ilpo Koskinen, John Zimmerman, Thomas Binder, Johan Redström, and Stephan Wensveen, Design
} Research Through Practice: From the Lab, Field, and Showroom (Waltham, MA: Elsevier, 2011). 
of one-dimensional idealized objects, including knots, links, braids, and tangles ${ }^{15}$ that are made up of infinite thread and can be continuously deformed without breaking. ${ }^{16}$ By definition a mathematical knot is a "closed loop in three-dimensional space" which has "no free ends." 17

The mathematical study of knots has a focus on properties that relate to the positions of threads in space, ${ }^{18}$ the patterns of knots, and the number of crossings. It is not concerned with physical properties, such as tension, size, and the shape of individual loops. ${ }^{19} \mathrm{~A}$ fundamental problem in knot theory is determining whether two knots are equivalent. Solving this involves seeking a property of each knot that does not change when the knot is subjected to manipulations. For each property, for example the number of crossings, a knot invariant may be defined. ${ }^{20}$ Knot diagrams are employed as mathematical tools to illuminate knot properties and determine equivalence, if it exists. A knot diagram is a projection or representation of a mathematical knot using simple line drawings to indicate the knot pattern. Lines in the diagram are broken to show where the knot crosses itself (Figure 2). The use of diagrams makes problem solving highly visual.

\footnotetext{
${ }^{15} \mathrm{~A}$ link is a combination of several knots inter-chained together; a braid is a set of ascending threads with end points fixed on two parallel lines, one under the other; and a tangle is an arbitrary set of threads with fixed end points. See Colin Adams, The Knot Book: An Elementary Introduction to the Mathematical Theory of Knots (New York, NY: W.H. Freeman, 1994).

${ }^{16}$ Alexei Sossinsky, Knots: Mathematics with a Twist (Cambridge, MA: Harvard University Press, 2002).

${ }^{17}$ Keith Delvin, The Language of Mathematics: Making the Invisible Visible (New York, NY: W.H. Freeman, 1998): 248-49. A plane closed curve and a knot are topologically equivalent. See H. Graham Flegg, From Geometry to Topology, (Mineola, NY: Dover Publications, 2001): 17-19.

${ }^{18}$ Adams, The Knot Book.

${ }^{19}$ Delvin, The Language of Mathematics.

20 ibid.
} 


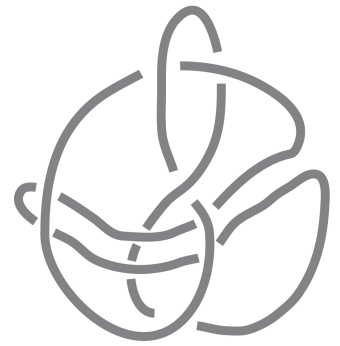

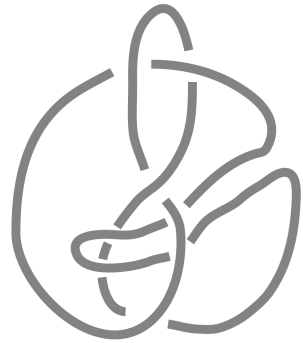

b

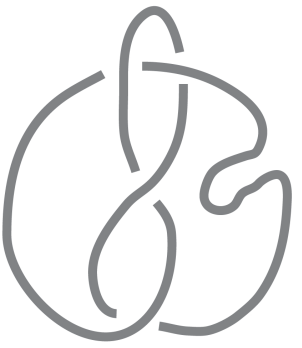

c

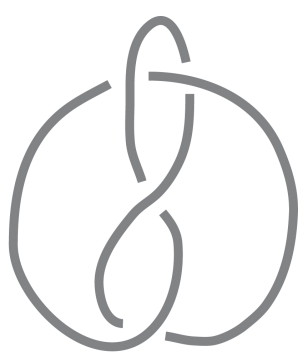

Figure 2. Mathematical knot diagrams illustrate crossings. The knots in all diagrams are equivalent representations of the same knot as the loop in a may gradually be removed ( $b$ and $c$ ) to show a representation of the figure-eight knot in diagram $d$. ( ) Janette Matthews.

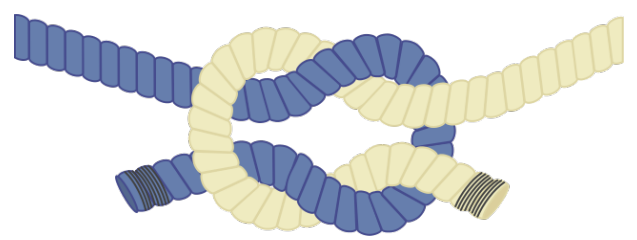

Figure 3. Drawing of a reef or square knot that depicts the knot's physical properties $\odot$ Creative Commons. $^{21}$

Drawings may also be used to depict a physical craft knot made from string or any other material. However, unlike a mathematical knot diagram, the illustration of a craft knot attempts to provide a realistic representation, using continuous bordered and/or textured lines, perhaps even shaded, to depict loose or tight knots, and may include additional arrows to demonstrate the knotting process (Figure 3 ). ${ }^{22}$ While a craft knot illustration aims to reveal the physical properties of a knot and perhaps the process for knotting, a mathematical knot diagram focuses on the pattern and the position of strands. This differentiation can be seen elsewhere in textiles. Weaving illustrations also use simple lines, similarly to mathematical knot diagrams, to show

\footnotetext{
${ }^{21}$ Wikimedia Commons, accessed May 13, 2016,

<https://commons.wikimedia.org/wiki/File:Reef_knot_noeud_plat.svg> .

${ }^{22}$ For more craft knots, see, for example, Clifford W. Ashley, The Ashley Book of Knots (London: Faber and Faber, 1993).
} 
the position of warps and wefts (vertical and horizontal threads) whereas a weaving plan will indicate how the loom is to be configured and the order in which warp yarns are to be raised. Schematics and three-dimensional representations can be seen in software for designing woven fabrics and computer-controlled looms, e.g., WeavePoint, ScotWeave, and WeaveMaker. ${ }^{23}$

\section{Research Through Design: A Broad Methodological Framing}

In design research, the utilization of professional design practice as a vehicle for research, an approach termed "practice-based research" or "research through design", has been under debate for over two decades. Most debates refer to Frayling's three distinctive models of research in art and design in $1993 .{ }^{24}$ One of the three models, "research through art and design," has been adopted by professional designers, because it provides them with opportunities to develop their design practice and to progress into $\mathrm{PhD}$ education. However, when design practice is used as a research methodology, experience within and from the practice has to be made explicit so that the embodied knowledge generated is transferable. The communication and transferability of embodied or tacit knowledge has raised an issue of how research through design should be disseminated when "we can know

\footnotetext{
${ }^{23}$ While ScotWeave offers CAD/CAM tools for dobby, jacquard, and 3D weaving, WeavePoint and WeaveMaker are CAD/CAM software for dobby weaving only. These programs are developed in different parts of the world: WeavePoint (Norway), ScotWeave (UK), and WeaveMaker (USA). See WeavePoint <http://www.weavepoint.com/>, ScotWeave, <http://www.scotweave.com/>, and WeaveMaker <http://www.weavemaker.com/>, accessed May 9, 2016.

${ }^{24}$ Christopher Frayling categorized research in art and design into three distinctive models: (1) research for art and design, (2) research into art and design, and (3) research through art and design. See Christopher Frayling, "Research in Art and Design," Royal College of Art Research Papers 1, no. 1 (1993): 1-5.
} 
more than we can tell"25 and when some of the knowledge is embodied in the design outputs or artifacts. A constructive role of designers in cross-disciplinary inquiry lies in the dissemination of tacit or embodied knowledge through the generation of artifacts together with the designers' ways of organizing knowledge from other disciplines to make and represent artifacts. ${ }^{26}$ It can be implied that spoken or written language alone may not be sufficient; knowledge of practice needs to also be demonstrated through artifacts and their process of making. ${ }^{27}$

\section{Collaborative Approach of Textile Knotting and Mathematical Knot Theory}

Following the research through design model to conduct cross-disciplinary collaborative research which is used here, Nimkulrat is a textile practitioner, while Matthews, although also a textile practitioner assumes the role of mathematician, analyzing Nimkulrat's textile practice, applying relevant theories and communicating insights. Through mathematical characterization of a single craft knot used in

\footnotetext{
${ }^{25}$ Michael Polanyi, Personal Knowledge: Towards a Post-Critical Philosophy (Chicago, IL: University of Chicago Press, 1958), 8.

${ }^{26}$ Chis Rust gives a number of research projects in which designers experiment and produce prototypes in collaboration with scientists in order to answer various research inquiries and demonstrate the findings for their research. See Chris Rust, "Design Enquiry: Tacit Knowledge and Invention in Science, Design Issues 20, no. 4 (2004): 80-82,

${ }^{27}$ Chris Rust's view on design artifacts in research inquiry is supported by Biggs's perspective on research in art and design which addresses creative practice as an integral part of not only the communication of research results but also the process of research. Practice is therefore understood as a research method and a means for communicating research findings. See Michael Biggs, "Approaches to the Experiential Component of Practice-Based Research, in Forskning - Reflektion - Utveckling [Research - Reflection - Development], ed. Henrik Karlsson (Stockholm, Sweden: Swedish Research Council, 2004), 6-21.
} 
Nimkulrat's work, ${ }^{28}$ the authors revealed differences between textile knot practice and mathematical knot theory (Table 1). The difference in "ends"29 was explored further using a knot diagram and its colorable property. ${ }^{30}$ The coloring of the diagram of Nimkulrat's knot after all ends were joined showed that the knot was composed of four trivial knots (by definition a knot with no crossings) tangled together (Figure 4b). This aspect stimulated new ideas, and supported the design and production of a new knot structure (Figure 5) that contains no loose ends. ${ }^{31}$ In order to construct knots without loose ends, it was necessary to use a material that is either already in a ring form or is thick enough for the ends to be joined seamlessly. Neoprene cord, which Nimkulrat would not have normally selected, became the material, as joining ends is achievable through bonding with adhesive.

\footnotetext{
${ }^{28}$ Nithikul Nimkulrat and Janette Matthews, "Mathematical Textiles: The Use of Knot Theory to Inform the Design of Knotted Textiles," in Proceedings of the 5th International Congress of International Association of Societies of Design Research (IASDR 2013) (Tokyo, Japan: Shibaura Institute of Technology, 2013): 1048-59.

${ }^{29}$ By definition mathematical knots consist of continuous line(s), whereas the tying of a craft knot utilizes one or more threads that have loose ends.

${ }^{30}$ Adam, The Knot Book.

${ }^{31}$ Nithikul Nimkulrat and Janette Matthews, "From Mathematical Diagrams to Knotted Textiles," in Proceedings of Bridges 2014: Mathematics, Music, Art, Architecture, Culture, ed. Gary Greenfield, George W. Hart, and Reza Sarhangi (Phoenix, Az: Tessellations, 2014): 219-24.
} 
"Ways of Being Strands: Cross-Disciplinary Collaboration using Craft and Mathematics"

Table 1. Differences between textile knot practice and mathematical knot theory ${ }^{32}$

\begin{tabular}{|c|c|c|}
\hline Property & Textile knot practice & Mathematical knot theory \\
\hline Ends & May have loose ends. & $\begin{array}{l}\text { Continuous curve with no loose } \\
\text { ends. }\end{array}$ \\
\hline Material & $\begin{array}{l}\text { Material dependent. The } \\
\text { appearance of a knot is } \\
\text { governed by material properties } \\
\text { and dimensions. }\end{array}$ & $\begin{array}{l}\text { Not concerned with materiality. Cross } \\
\text { section of strand deemed to be a } \\
\text { point. }\end{array}$ \\
\hline Tension & $\begin{array}{l}\text { Tension dependent. Internal and } \\
\text { external spaces are pertinent. }\end{array}$ & $\begin{array}{l}\text { A tight knot has the same } \\
\text { representation as a loose knot so are } \\
\text { considered equivalent. }\end{array}$ \\
\hline Form & $\begin{array}{l}\text { The addition of extra loops } \\
\text { changes the appearance of a } \\
\text { knot. }\end{array}$ & $\begin{array}{l}\text { If a knot may be simplified to the } \\
\text { same representation as another knot, } \\
\text { they are considered equivalent. }\end{array}$ \\
\hline
\end{tabular}

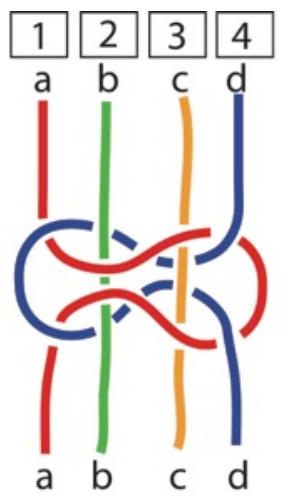

(a)

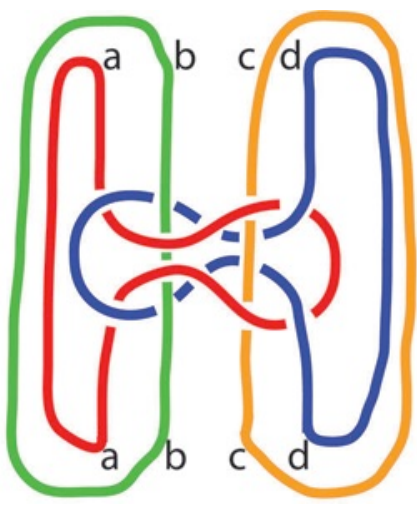

(b)

Figure 4. Diagram of a craft knot showing the position of strands (a). Four tangled trivial knots formed by joining all loose four ends of a craft knot (b). @ Janette Matthews.

\footnotetext{
${ }^{32}$ Nimkulrat and Matthews, "Mathematical Textiles," 7.
} 


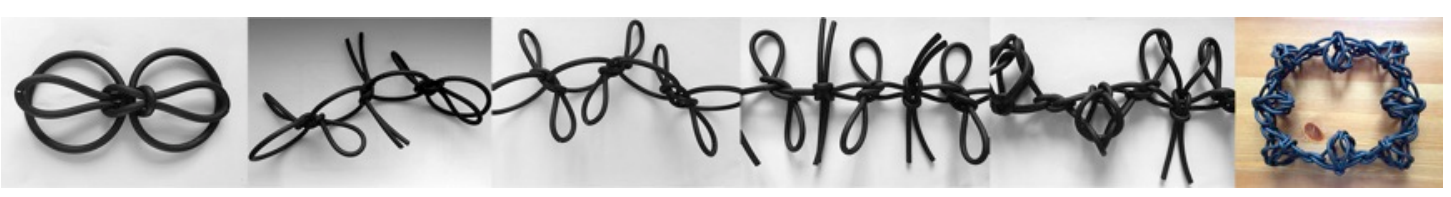

Figure 5. A new knot structure with no loose ends that have the starting point from Figure $4 \mathrm{~b}$. It is made of neoprene cord. (c) Nithikul Nimkulrat.

\section{Knotted Pattern Designs Influenced by Mathematics}

As stated above, a knot diagram's colorable property is used for studying the positions of strands in space. ${ }^{33}$ The coloring of the knot diagram of a single knot (Figure 4a) reveals that the positions of strands finish in the same place that they start, ${ }^{34}$ e.g., strand a starts and ends in position 1 . This property is not obvious in Nimkulrat's craft practice. The positions of strands in the diagram imply that there is the potential to design various knot patterns by changing the positions of strands.

To verify this, Matthews utilized the same method to characterize a group of knots in Nimkulrat's work (Figure 6) starting with four knots, each requiring four strands. Figure 7 shows that there are potentially four knots on each row and the remaining strands may be used to tie an additional knot which would result in a tubular structure as shown in Figure 6b. All knots are tied identically and each knot contains two active and two passive strands. The first and third rows of knots (Figure 7 , from top) use strands $a$ (red) and $d$ (blue) for knotting, while strands $b$ (green) and $c$ (yellow) play no role and are passive. The knots on the second and fourth rows have as active strands $b$ (green) and $c$ (yellow). The active strands from the previous row, a (red) and $d$ (blue), are passive. Again, all strands finish in the same positions in which they started.

\footnotetext{
${ }^{33}$ Adam, The Knot Book.

${ }^{34}$ Nimkulrat and Matthews, "Mathematical Textiles."
} 

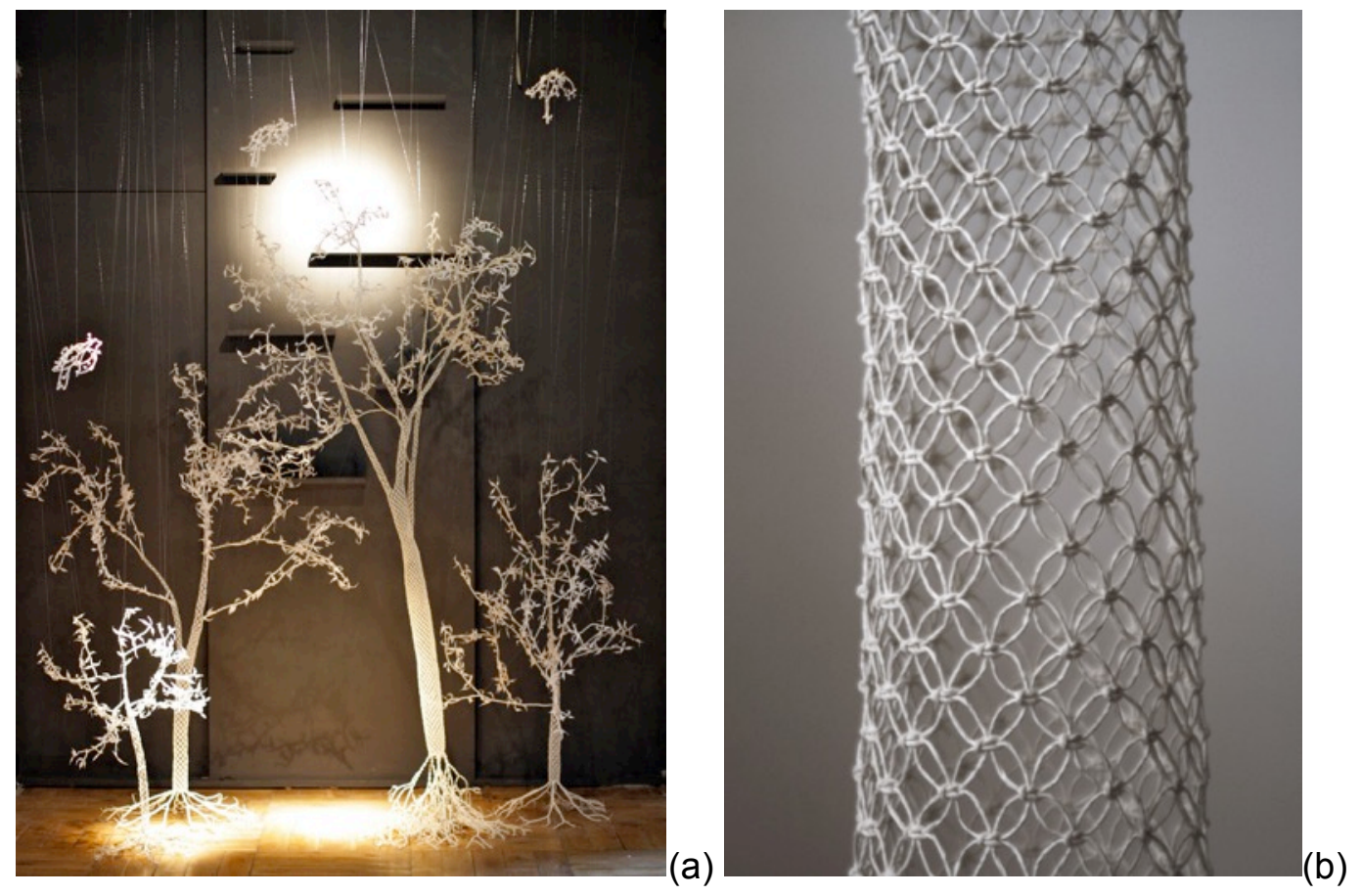

Figure 6. "The White Forest" (2012), dimensions: $150 \mathrm{~cm}$ x $100 \mathrm{~cm}$ x $200 \mathrm{~cm}$, material: paper string (a).

The structure of an individual piece from the installation made from the same knot used in repeats (b). ( Nithikul Nimkulrat.

Characteristics (e.g., start/end positions, active/passive roles) indiscernible through observing the work (Figure 6b) become evident in Figure 7. Repeating patterns became apparent-each knot on the same row was the same, alternate rows were the same. Recognizing this suggested two potential approaches for generating repeating knot pattern designs: (1) through the use of color and (2) through changing which strands are active and which are passive. 


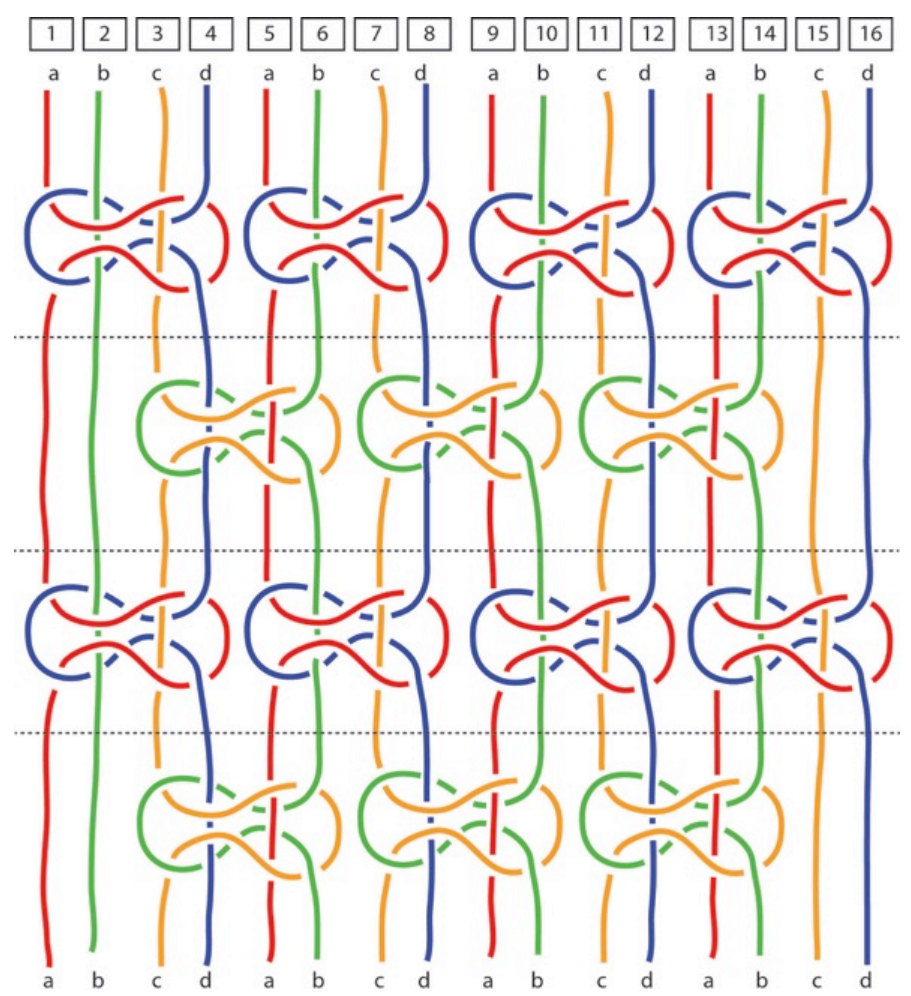

Figure 7. Diagram of four rows of knots showing rows one and two (from top) are repeated. ( Janette Matthews.

To explore the use of color, the number of colors was reduced to two to focus on the pattern generated from two different colors rather than the influence of colors themselves. To do this, Matthews re-colored Figure 7 with black and gray—gray replaced red and blue (the initial active strands), and black replaced green and yellow (the initial passive strands) (Figure 8). On examining Figure 8, the authors observed that a black knot is created when the strands tying it are black and a gray knot results when the active strands are gray. A further insight is that the strands linking the knots would appear to form either black or gray rounded rectangles-not a mixture of black and gray. 


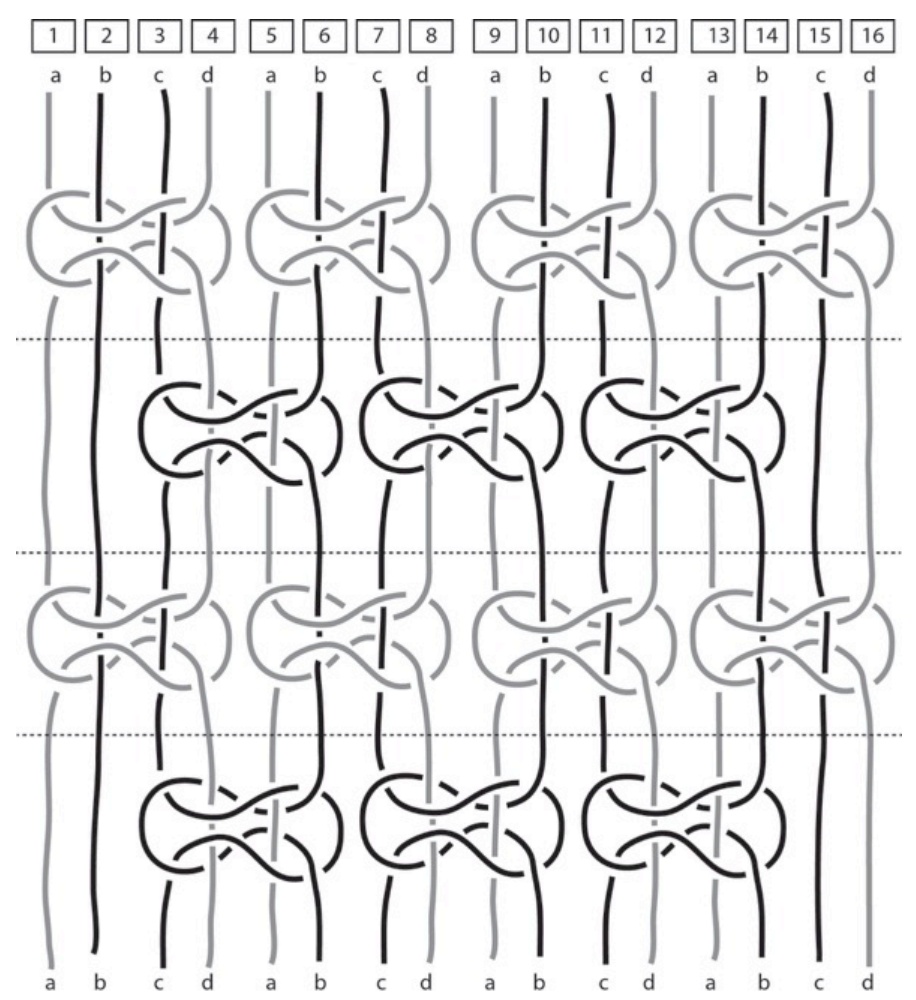

Figure 8. Diagram of four knots using two colors. (c) Janette Matthews.

To confirm this design possibility, Nimkulrat followed Figure 8 in knotting paper string, ordering the colors of the strands from left to right-white-black-blackwhite. The knotting started with using white string for the active strands on the first row (gray) and black string for the passive middle strands (black). A white knot was created, while the passive black strands were concealed (Figure 9a). Knots tied with the active black strands and the passive middle white strands were black (Figure 9b). White and black strands alternately played an active role in the tying of knots on alternate rows. Intertwining black and white circles became apparent (Figures 9c and $9 \mathrm{~d})$. The properties of paper string transformed rounded rectangles in the diagram into circles in the knotting (Figure 9d). 

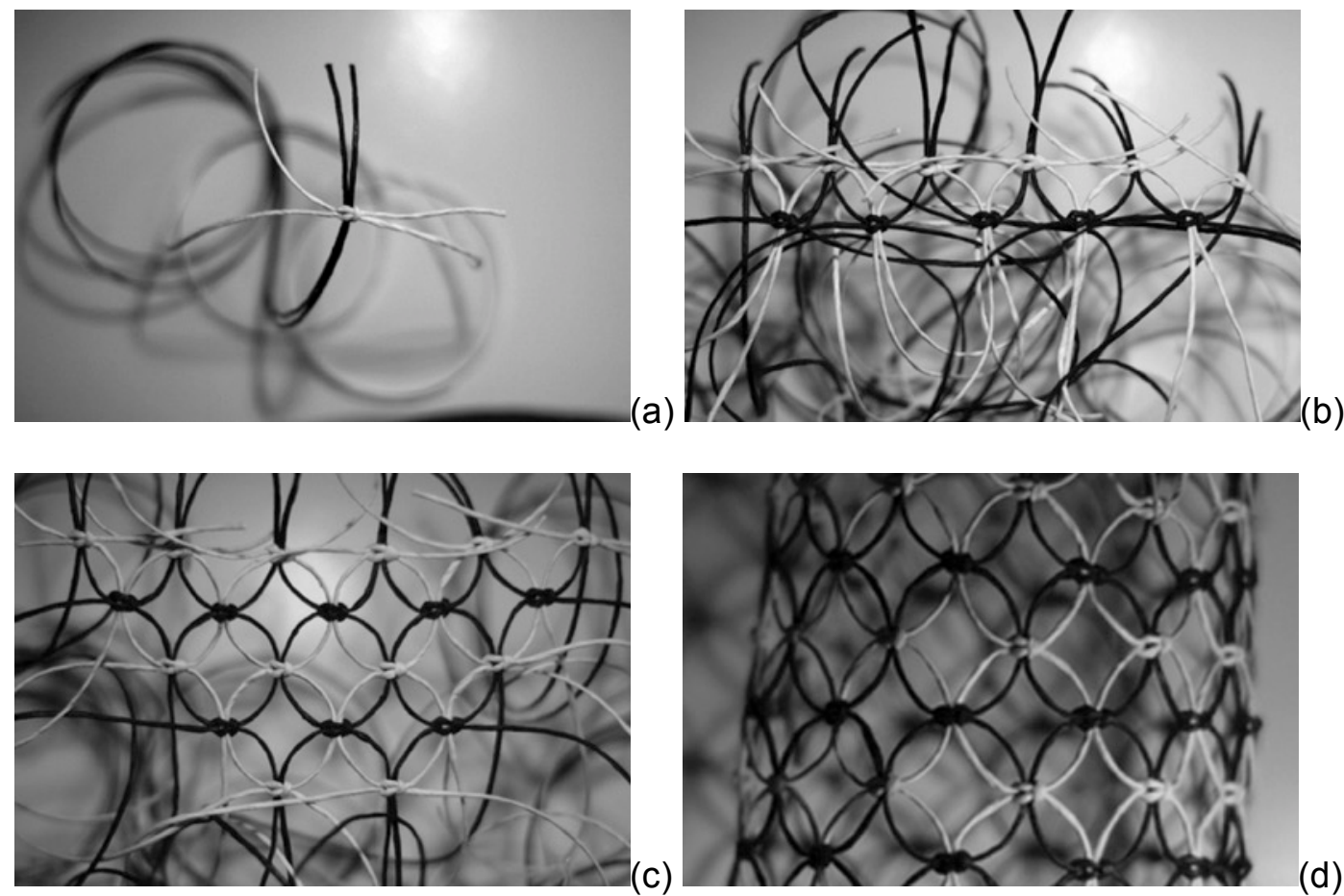

Figure 9. The knotting process using black and white paper string to create a circle pattern. ( Nithikul Nimkulrat.

Nimkulrat then experimented with other color combinations, for example positioning black and white strands: (from left to right) black-black-white-white (Figure 10a). Here both black and white strings simultaneously played a direct role in tying the knot. To link individual knots, one knot was flipped before the tying took place (Figure 10b), allowing all four strings of the same color to form a pure color knot, black or white (Figure 10c). The process continued alternately between a row of mixed color knots and that of pure black and white ones, leading to a striped knot pattern (Figure 10d). 

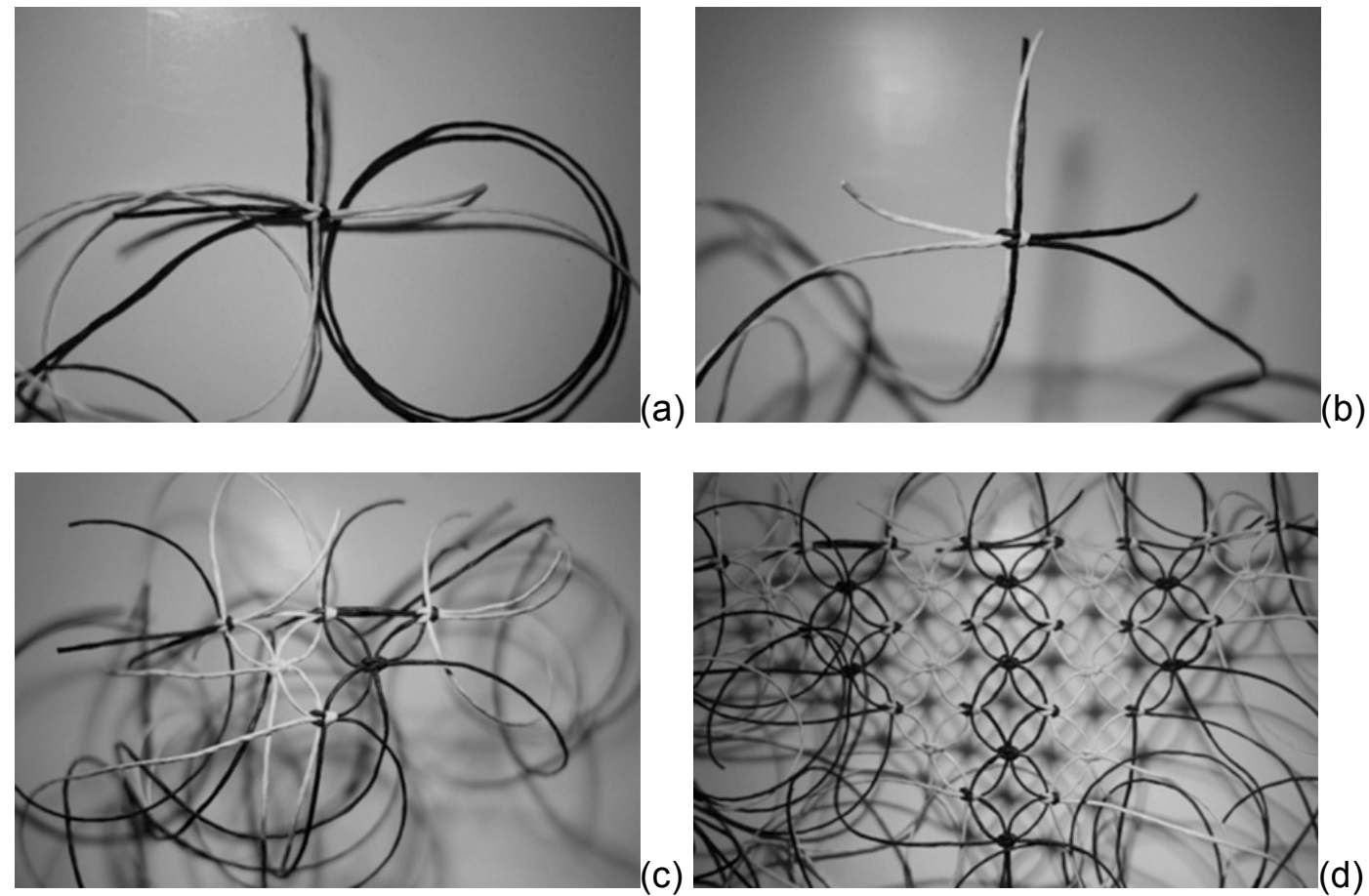

(d)

Figure 10. The knotting process using black and white paper strings in a different position of strands. (C) Nithikul Nimkulrat.

Following Nimkulrat's knotting, Matthews examined the knotted sample from Figure $10 \mathrm{~d}$ to determine whether this pattern could have been predicted from a colored diagram. Figure 11 shows the diagram colored on the basis of the starting position described above, $\operatorname{black}(a)$-black(b)-white $(c)$-white $(d)$, and then in the opposite order, white $(d)$-white $(c)$-black(b)-black(a), with white replaced with gray. It can be clearly seen that the pattern shown in Figure 10d emerges. 


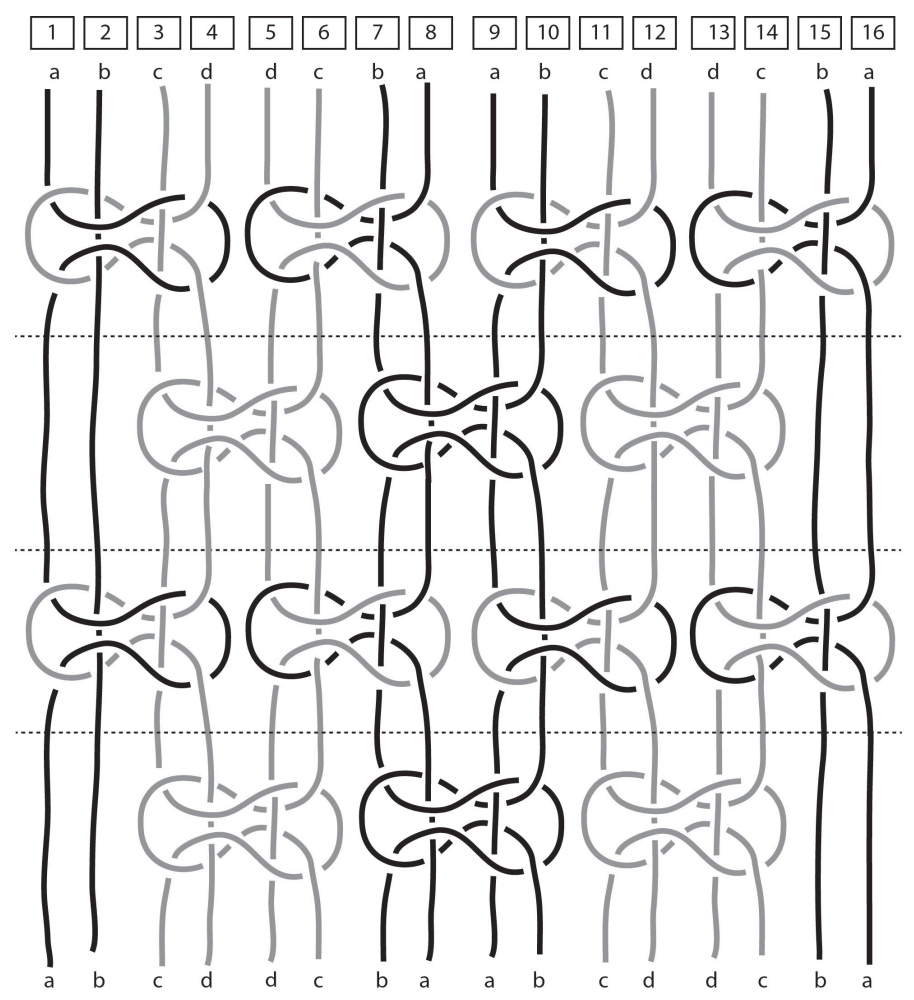

Figure 11. Diagram with two colors and alternate flipped knots on row one. () Janette Matthews.

Nimkulrat's new understanding of the positions of color strands in relation to pattern inspired new creation. Stripes, a basic pattern for interior textiles, e.g., curtains, upholstery, etc., inspired a functional form typically found in interiors-an armchair (Figure 1). While hand knotting the seat of this artifact, Nimkulrat observed that the stripe pattern was continuously created on all sides of the seat (Figures $1 \mathrm{a}$ and c). Although the knot diagrams did not facilitate the design of the threedimensional artifact, the stripe pattern had its origin in the diagrams and the design was provoked by experimentation based on the diagrams.

Re-coloring the diagram in different ways showed that a variety of design outcomes could be achieved (Figures 8 and 10). This approach provided, prior to making, a way of visualizing two-tone knot patterns. It is worth noting that prior to this cross-disciplinary collaboration, Nimkulrat had only created monochrome knotted textiles. She observed that she had wished to incorporate colors in her textile knot practice but had not attempted this. Even though she is a skillful practitioner of many 
years standing, the task of determining what strands should be used in which combinations to achieve different pattern combinations had seemed too difficult to realize. The diagrammatic approach offered a way forward. It allowed for experimentation and visualization prior to time-consuming knotting.

\section{Conclusion: Collaboration as a Cross-Disciplinary Approach in Research Through Design}

This article has elucidated cross-disciplinary collaboration between a mathematician and a designer as a research through design approach. An iterative process was developed between the two authors (Figure 12) that included initiating discussion to set goals, working individually, working together, articulating interesting observations, questioning, and setting goals for the next iteration. Within this collaboration, pattern development for knotted structures was explored, predicted, and modeled through a mathematical characterization process using knot diagrams. Diagramming textile techniques in color to show a process is not new. What is newly reported here is the application of the characteristics of mathematical knot diagrams to describe, interrogate, and then visualize craft knots. The diagrams are not graphical illustrations of textile knots but mathematical representations, which illuminate the textile process, provide insights into the nature of the textile craft and stimulate new ideas. Embedded in the diagrams are knotting characteristics, which cannot be observed from the work alone. Coloring the diagram makes the positions and roles of strands in a knotted structure explicit, and leads to an exploration of knotted pattern designs that may not have occurred otherwise. 
charaterized multiple knots diagrammatically using 4 colours.

recognized from the diagram of multiple knots the repeats that suggests the potential to generate repeating knot pattern designs.

re-colouring the diagram of multiple knots using 2 colours, black and gray.

observed that a black knot is created when the strands tying it are black

followed the black and grey diagram of multiple knots in knotting black and white paper string.

experimented with the positions of black/white strands to seek new patterns. A stripe knot pattern was created.

examined the knotted sample whether its stripe pattern could have been predicted from a coloured diagram.

\section{utilized the stripe knot pattern to make an artifact.}

Figure 12. Collaborative process between the authors presented in this article. ( Nimkulrat Nimkulrat.

The authors invested time and effort to share and integrate insights from both perspectives - textile craft and mathematics. In this collaboration, craft knot practice has on the one hand gained insights into the designing of new patterns through the mathematical knot diagrammatic method. On the other hand, through the artifact and samples exhibited in an exhibition together with dissemination, the diagramming methods of mathematical knot theory have been made available to a general audience. There have been a number of examples how textile crafts have an important role to play in illustrating mathematics to audiences, ${ }^{35}$ who may or may not

\footnotetext{
${ }^{35}$ See, for example, Taimina, Crocheting Adventures with Hyperbolic Planes and Harris, Common
}

Threads. 
have received formal mathematical education but are nonetheless interested. Initiatives (e.g., the Museum of Mathematics ${ }^{36}$ and the David and Claudia Harding Gallery ${ }^{37}$ ) are examples of an appetite to explore the creativity of mathematics through hands-on activities. The annual international Bridges ${ }^{38}$ conferences demonstrate the positive contributions arising from cross-disciplinary artmathematics research from the perspectives of both disciplines.

To summarize, this cross-disciplinary collaboration has enabled learning through reflection of processes and outcomes. Firstly, the collaborative practice empowered the team to embrace a fluid iterative process that aimed to tackle the research questions initially set. Through this approach, the researchers coordinated frequently and consistently, informing each other's work, so that individual control over the outcome was released and the chance to explore the range of potentials generated from the researchers' integration of insights emerged. Secondly, the success of collaboration required the development of a communication strategy or a common language between the researchers. In the collaboration discussed in this article, the authors found a common language to communicate research findings and novel ideas through the use of test samples, colored knot diagrams, photographs, and artifacts. Thirdly, individual accountability was found to be a supportive attribute of integrated work and the means by which researchers depended on each other in

\footnotetext{
${ }^{36}$ National Museum of Mathematics, accessed May 11, 2016, http://momath.org/.

${ }^{37}$ David and Claudia Harding Gallery, Science Museum, London opening in 2016, accessed May 12, 2016, http://www.sciencemuseum.org.uk/visitmuseum/new_galleries/mathematics_gallery

${ }^{38}$ The Bridges Organization was formed in 1998 to foster research, practice, and new interest in mathematical connections to art, music, architecture, education, and culture. See http://bridgesmathart.org. The Bridges organizes annual conferences with exhibitions in which the authors presented this ongoing project in 2014 (paper only) and 2016 (paper and artifacts in the exhibition).
} 
the collaboration. A strategy that clarified timelines, requirements, and responsibilities was important to generate effective interaction and a working rhythm between the collaborators.

It is interesting to offer a personal comment on the respective roles of mathematics and artifacts in this ongoing study. The application of mathematical knot diagrams and other mathematical methods by Matthews provided a disciplined framework through which to analyze and develop Nimkulrat's craft practice, e.g., how the work was knotted, what influenced pattern, what the role of materiality was, and how ideas might be generated or tested. The diagrams used to communicate mathematical insights to Nimkulrat became in themselves a tool for design development and prediction. Reflecting on the research process, the impact of mathematics on craft practice is clear. The samples and artifacts highlighted the opportunities that might arise from the use of mathematics in textile design research. The question arises as to what extent this study or elements of craft practice can influence, inform, or communicate mathematics. From the authors' observation of audience $^{39}$ engagement with and responses to the artifacts at conferences, it became apparent that the audience's understanding of how craft practice could influence, inform, or communicate mathematics depended on their prior knowledge of mathematics. Not all mathematical concepts or topics are inaccessible to those outside the discipline. Further research will seek to identify further craft practice and will examine threshold concepts that are needed to enable non-mathematically trained textile/art practitioners to access mathematical concepts with the explicit aim of cross-disciplinary enquiry.

\footnotetext{
${ }^{39}$ The samples and artifacts produced within this collaboration were exhibited in the RTD 2015 Exhibition and "Room of Interest" in Cambridge, UK and the Bridges 2016 Exhibition in Jyväskylä, Finland. The audiences were participants in these conferences who could experience the physical artifacts and converse with the authors.
} 


\section{Bibliography}

Adams, Colin. The Knot Book: An Elementary Introduction to the Mathematical Theory of Knots. New York, NY: W.H. Freeman, 1994.

Adams, Robin. "Cross-Disciplinary Ways of Thinking, Acting, and Being," Lecture at Tufts University, Medford, MA, December 6, 2010. http://sites.tufts.edu/tuftsstemlecture/previous-lectures/cross-disciplinaryways-of-thinking-acting-and-being/

Ashley, Clifford W. The Ashley Book of Knots. London: Faber and Faber, 1993.

Belcastro, Sarah-Marie, and Yackel, Carolyn, ed. Making Mathematics with Needlework: Ten Papers and Ten Projects. Natick, MA: A K Peters, 2009.

—_. Crafting by Concepts: Fiber Arts and Mathematics. Natick, MA: A K Peters, 2011.

Biggs, Michael. "Approaches to the Experiential Component of Practice-Based Research. In Forskning - Reflektion - Utveckling [Research - Reflection Development], edited by Henrik Karlsson, 6-21. Stockholm, Sweden: Swedish Research Council, 2004.

Croom, Fred H. Principles of Topology. Mineola, NY: Dover Publications. 2016.

Delvin, Keith. The Language of Mathematics: Making the Invisible Visible. New York, NY: W.H. Freeman, 1998.

Feijs, Loe M. G. "Geometry and Computation of Houndstooth (Pied-de-Poule)." In Bridges 2012: Mathematics, Music, Art, Architecture, Culture, edited by Robert Bosch, Douglas McKenna, and Reza Sarhangi, 299-306. Phoenix, Az: Tessellations, 2012.

Feijs, Loe M. G., and Marina Toeters. "Constructing and Applying the Fractal Pied de Poule (Houndstooth)." In Bridges 2013: Mathematics, Music, Art, Architecture, Culture, edited by George W. Hart and Reza Sarhangi, 429-32. Phoenix, Az: Tessellations, 2013. 
Feijs, Loe M. G., Marina Toeters, Jun Hu, and Jihong Liu. "Design of a Nature-Like Fractal Celebrating Warp Knitting." In Bridges 2014: Mathematics, Music, Art, Architecture, Culture, edited by Gary Greenfield, George W. Hart, and Reza Sarhangi, 369-72. Phoenix, Az: Tessellations, 2014.

Flegg, H. Graham. From Geometry to Topology. Mineola, NY: Dover Publications, 2001.

Frayling, Christopher. "Research in Art and Design." Royal College of Art Research Papers 1, no. 1 (1993): 1-5.

Gray, Carole, and Gordon Burnett. "Making Sense: An Exploration of Ways of Knowing Generated Through Practice and Reflection in Craft." In Crafticulation and Education, edited by L. K. Kaukinen, 44-51. Helsinki: NordFo, 2009.

Harris, Mary. "Common Threads: Mathematics and Textiles." Mathematics in School 17 , no. 4 (1988): $24-28$.

Harris, Mary. Common Threads: Women, Mathematics and Work. Stoke-on-Trent: Trentham Books, 1997.

Isaksen, Daniel C., and Alabama P. Petrofsky. "Mobius Knitting." In Bridges 1999: Mathematical Connections in Art, Music, and Science, edited by Reza Sarhangi, 67-76. Arkansas, KS: Gilliland, 1999.

Koskinen, Ilpo, John Zimmerman, Thomas Binder, Johan Redström, and Stephan Wensveen. Design Research Through Practice: From the Lab, Field, and Showroom. Waltham, MA: Elsevier, 2011.

Nimkulrat, Nithikul. "Material inspiration: From Practice-Led Research to Craft Art Education." Craft Research 1, no. 1 (2010): 63-84.

—_. "Hands-on Intellect: Integrating Craft Practice into Design Research." International Journal of Design 6, no. 3 (2012): 1-14.

Nimkulrat, Nithikul, and Janette Matthews. "Mathematical Textiles: The Use of Knot Theory to Inform the Design of Knotted Textiles." In Proceedings of the 5th 
International Congress of International Association of Societies of Design Research (IASDR 2013), 1048-59. Tokyo, Japan: Shibaura Institute of Technology, 2013.

. "From Mathematical Diagrams to Knotted Textiles." In Proceedings of Bridges 2014: Mathematics, Music, Art, Architecture, Culture, edited by Gary Greenfield, George W. Hart, and Reza Sarhangi, 219-24). Phoenix, Az: Tessellations, 2014.

—_. "Ways of Being Strands: Exploration of Textile Craft Knots by Hand and Mathematics." In Proceedings of the 2nd Biennial Research Through Design Conference, 25-27 March 2015, Cambridge, UK, Article 14. DOI: 10.6084/m9.figshare.1327996.

Osinga, Hinke M., and Bernd Krauskopf. "Crocheting the Lorenz Manifold." The Mathematical Intelligencer 26, no. 4 (2004): 25-37.

_ . "How to Crochet a Space-Filling Pancake: The Math, The Art and What Next. In Proceedings of Bridges 2014: Mathematics, Music, Art, Architecture, Culture, edited by Gary Greenfield, George W. Hart, and Reza Sarhangi, 1926. Phoenix, Az: Tessellations, 2014.

Polanyi, Michael. Personal Knowledge: Towards a Post-Critical Philosophy. Chicago, IL: University of Chicago Press, 1958.

Rust, Chris. "Design Enquiry: Tacit Knowledge and Invention in Science. Design Issues 20, no. 4 (2004): 77-85.

Sennett, Richard. The Craftsman. New Haven, CT: Yale University Press, 2008. Sossinsky, Alexei. Knots: Mathematics with a Twist. Cambridge, MA: Harvard University Press, 2002.

Taimina, Diana. Crocheting Adventures with Hyperbolic Planes. Wellesley, MA: AK Peters, 2009. 